\title{
Elaboración de una prueba diagnóstico para medir habilidades de escritura académica
}

\section{Development of a diagnostic test to measure academic writing skills}

\author{
Carmen Elena Muñoz Rodríguez ${ }^{1}$ \\ carmen.munoz@umich.mx \\ Bernardo Enrique Pérez Álvarez \\ bernardo.perez@umich.mx \\ Universidad Michoacana de San Nicolás de Hidalgo, México
}

\begin{abstract}
Resumen:
El objetivo de esta investigación consistió en aplicar un instrumento diagnóstico que permita determinar el grado de alfabetización académica alcanzado por estudiantes universitarios respecto a su nivel de estudios (pregrado o posgrado), es decir, se analizaron los conocimientos, habilidades, destrezas, creencias y/o prejuicios que posee el estudiante en cuanto al proceso de escritura. Para ello, en la metodología se describe el proceso de elaboración de un instrumento diagnóstico para la evaluación de las habilidades de escritura de estudiantes (de pregrado y posgrado), quienes acuden al Centro de Escritura de la Universidad Michoacana de San Nicolás de Hidalgo con el propósito de obtener ayuda en sus productos escritos. Los resultados obtenidos fueron abordados desde un análisis cualitativo, con una correlación entre las características principales que los estudiantes consideran son
\end{abstract}

\begin{abstract}
:
This study aimed to apply a diagnostic instrument that allowed determining the degree of academic literacy achieved by students in relation to their level of studies (undergraduate or graduate). That is, students' knowledge, abilities, skills, beliefs and / or prejudices regarding the writing process were analyzed. The methodology describes the process of elaborating a diagnostic instrument for the evaluation of students' writing skills who visit the Writing Center of the Universidad Michoacana de San Nicolás de Hidalgo with the purpose of getting help on their written products. The results obtained were analyzed from a qualitative perspective, with a correlation between the main characteristics that the students consider are important in the task of writing. The results found that students work (i) to develop those areas that allow a text to be coherent, (ii) to have a good command of spelling; and (iii) to
\end{abstract}

\section{Dirección para correspondencia (correspondence address):}

Carmen Elena Muñoz Rodríguez. Universidad Michoacana de San Nicolás de Hidalgo. Instituto de Investigaciones Filosóficas "Luis Villoro Toranzo" Edificio C-4A, Ciudad Universitaria. Avenida Francisco J. Múgica S/N. C.P. 58030 Morelia, Michoacán (México). 
importantes en el quehacer de la escritura. De esta manera, se trabajó para mostrar que las habilidades que deben desarrollar son aquellas que permitan que un texto sea coherente, tener un buen manejo de la ortografía y cómo comenzar a redactar. El examen diagnóstico permitió establecer los parámetros para delimitar las características que debe cumplir el usuario en el proceso de escritura para considerar que desarrolla bien esta actividad, y que ha alcanzado un nivel de suficiencia en la alfabetización académica que le permita desarrollarse en el ámbito universitario.

\section{Palabras clave:}

Escritura académica; centro de escritura; diagnóstico de escritura; habilidades de escritura. learn how to start writing. The diagnostic test made it possible to establish the parameters which define the characteristics that users must meet in the writing process to consider that they develop this activity well, and that they have reached a level of sufficiency in academic literacy that allows them to get by in the university environment.

\section{Key words:}

Academic writing; writing center; writing diagnosis; writing skills.

\section{Résumé:}

L'objectif de cette recherche était de mettre en pratique un outil de diagnostic permettant de déterminer le degré d'écriture académique atteint par des étudiants universitaires en fonction de leur niveau d'études (premier cycle ou diplômé), c'est-à-dire qu'ils ont analysé les connaissances, les capacités, les compétences, les croyances et / ou les préjugés que l'étudiant a sur le processus d'écriture. Pour cela, la méthodologie décrit le processus d'élaboration d'un outil de diagnostic pour l'évaluation des compétences en écriture des étudiants (premier cycle et diplômé), qui se rendent au Centre d'écriture de I'Université Michoacana de San Nicolás de Hidalgo dans le but d'obtenir de l'aide pour leurs productions écrites. Les résultats obtenus ont été analysés à partir d'une perspective qualitative, avec une corrélation entre les principales caractéristiques que les étudiants considèrent comme importantes dans la tâche d'écriture. De cette manière, ils ont travaillé pour montrer que les compétences à développer sont celles qui permettent à un texte d'être cohérent, d'avoir une bonne maîtrise de l'orthographe et de commencer à écrire. Le test diagnostique a permis d'établir les paramètres pour délimiter les caractéristiques que l'utilisateur doit respecter dans le processus d'écriture pour considérer qu'il réalise correctement cette activité, et qu'il a atteint un niveau suffisant en écriture académique pour se développer en milieu universitaire.

\section{Mots clés:}

Écriture académique, centre d'écriture, diagnostic d'écriture, compétences en écriture.

Fecha de recepción: 22-10-2020

Fecha de aceptación: 28-01-2021 


\section{Introducción}

Leer y escribir son actividades estratégicas que permiten el desarrollo y la apertura al mundo en el ámbito universitario; "la educación universitaria requiere que los alumnos tengan un dominio mínimamente bueno de las habilidades de comprensión de lectura, redacción y expresión oral" (Guerra y Pérez, 2017, p. 162), por lo anterior, en las últimas décadas se ha llevado a cabo una profunda reflexión acerca de estas prácticas, asumiendo que deben ser atendidas y promovidas, pues es a través de ellas como los estudiantes acceden y se apropian del conocimiento (Bitonte y Lo Coco, 2013; Carlino, 2005; Cassany, 2015). Esta labor es el cimiento sobre el que se basa la creación de Programas y Centros de Escritura encaminados a desarrollar la alfabetización académica, atendiendo las deficiencias en las habilidades de escritura a través del apoyo a los usuarios en la elaboración de textos académico-científicos desde el autoaprendizaje (Roldán, Cárdenas y Casanova, 2013).

El Centro de Escritura (CE) de la Universidad Michoacana de San Nicolás de Hidalgo (UMSNH) surgió en agosto de 2018 como un programa cuya finalidad es colaborar en el desarrollo de las habilidades de escritura en la comunidad universitaria, brindando a los estudiantes (de licenciatura y posgrado), docentes, investigadores y administrativos atención en sus necesidades específicas de escritura. El CE además de apoyar a los usuarios en la elaboración de documentos específicos, también busca proporcionar estrategias para facilitar el autoaprendizaje en relación a la redacción, las habilidades de escritura y en la referenciación de documentos, estas actividades se desarrollan a través de los diferentes servicios que en el centro se ofrecen y que se agrupan bajo tres modalidades: asesorías individuales, asesorías grupales y talleres.

Uno de los pasos necesarios en la implementación del CE en la UM$\mathrm{SNH}$ fue la elaboración de un instrumento diagnóstico que permitiera conocer la situación actual en la que llegan los usuarios en sus habilidades de lectura y escritura, pues con este primer acercamiento se pretende hacer más eficientes los servicios que en el centro se ofrecen. Asimismo, se busca conocer qué dimensiones o niveles de la actividad de escritura son más urgentes de atender tanto a nivel individual como a nivel institucional; "diagnosticar a los usuarios permite, de un modo individual, encauzar el trabajo en las tutorías personales, pues rentabiliza las sesiones y permite orientar el tratamiento autónomo de los usuarios" 
(Martínez y Llamas, 2015, p. 25). El CE está enfocado en el proceso de producción del texto y en el escritor mismo, más que en el producto textual, se trata por lo tanto de un enfoque procesual (Cassany, 2007), y constituye un instrumento útil para conocer cuáles son las habilidades y debilidades que los usuarios poseen.

El objetivo de la investigación es aplicar un instrumento diagnóstico que permita determinar el grado de alfabetización académica alcanzado por los usuarios respecto a su nivel de estudios (pregrado o posgrado), es decir, se trata de determinar los conocimientos, habilidades, destrezas, creencias y/o prejuicios que posee el estudiante en cuanto al proceso de escritura, y a través de las asesorías, favorecer la mejora y desarrollo de la escritura crítica y epistémica. De igual forma, favorecer la autorregulación del proceso de escritura para alcanzar las competencias esperadas de acuerdo al nivel y plan de estudios.

Para ello, se describe el proceso de elaboración de un instrumento diagnóstico para la evaluación de las habilidades de escritura de estudiantes (de pregrado y posgrado), quienes acuden al CE de la UMSNH con el propósito de obtener ayuda en sus productos escritos, los resultados obtenidos hasta ahora han sido abordados desde un análisis cualitativo.

También se describirán algunos aspectos importantes de la prueba diagnóstica, como el contexto en que se aplica, los ejercicios propuestos, los parámetros que pueden medirse, así como algunos de los resultados obtenidos hasta el momento. Con esta información, al final se plantean algunas reflexiones acerca de los posibles alcances que puede tener esta prueba así como los aspectos en que puede mejorarse para lograr un instrumento diagnóstico de amplio alcance en el ámbito universitario.

\section{Marco empírico}

De los tres servicios que en el CE se ofrecen, dos están centrados en las situaciones concretas de los usuarios. Tanto en las asesorías individuales como en las asesorías grupales, la sesión se centra en la resolución de necesidades específicas de escritura, por lo que es preciso que los usuarios presenten un borrador del texto que desean revisar para trabajarlo entre pares. De esta forma el compañero de escritura durante la asesoría transmite información a los usuarios, recomendado, explicando y ejem- 
plificando (Molina, 2019). El compañero de escritura colabora con el usuario para revisar el texto al mismo tiempo que le brinda herramientas que puede desarrollar cuando se enfrente al quehacer de la escritura, pues en las asesorías lo que se pretenden es "Fomentar conductas autorregulativas, que permitan al aprendiz guiar autónomamente su proceso de aprendizaje y composición" (Cassany, 2015, p. 210).

Para brindar la asesoría entre pares es necesario conocer las habilidades y/o debilidades de escritura de los usuarios, si bien los usuarios presentan un borrador del texto que desean revisar, éste no es suficiente para evaluar su capacidad escritora, es por ello que al iniciar la sesión es necesario aplicar una prueba diagnóstica a través de la cual se "explora la capacidad actual del aprendiz, para identificar necesidades y programar objetivos de enseñanza" (Cassany, 2015, p. 250), en otras palabras, se trata de obtener un diagnóstico del grado de aprendizaje esperado en los estudiantes universitarios en relación a la alfabetización académica de los diferentes niveles (pregrado y posgrado) (Guzmán-Simón y García-Jiménez, 2015).

El modelo de las asesorías individuales tiene una duración de 45 a 60 minutos; en la fase inicial se indaga acerca de las ideas que el usuario necesita para comenzar o continuar el desarrollo de su texto, esto implica reflexionar sobre el motivo de la asesoría, el tipo de texto en el que se está trabajando y qué es lo que se ha adelantado; enseguida el usuario comienza a responder la prueba diagnóstica al tiempo que el compañero de escritura comienza a leer el borrador del usuario. Al finalizar cada asesoría, el asesor debe completar una ficha con los datos del usuario en la que se hace un pequeño informe de la labor realizada, asimismo se dan rasgos particulares acerca de las características del proceso de escritura que se observaron de manera individual. Durante este proceso los compañeros de escritura, a través de la prueba diagnóstica y de la revisión sistemática de casos específicos, evalúan el producto escrito, pero además (y más importante) evalúan el proceso y otros aspectos relacionados con la actividad de escribir en general aunque se parta de una situación concreta; se trata de una evaluación que cumple una función formativa o evaluación para el aprendizaje (Guzmán-Simón y García-Jiménez, 2015), ya que "se realiza en el proceso de aprendizaje y no al final de este, es decir, en el momento en el que pueden tomarse decisiones educativas para ajustar la enseñanza de acuerdo a las necesidades de los alumnos y realimentarlos durante su proceso de 
aprendizaje" (Sánchez y Martínez, 2020, p. 37); en síntesis, lo que se busca es hacer una valoración de las habilidades de escritura, así como una retroalimentación. A diferencia de otro tipo de evaluaciones, el objetivo de este paso no es puntuar o calificar el proceso o el producto de escritura.

La prueba diagnóstica que se ha formulado para el CE contiene tres ejercicios a través de los cuales se busca medir diferentes aspectos de las actividades lectora y escritora, el primero corresponde a un cuestionario (compuesto por siete preguntas) de concepciones en torno a la escritura; este cuestionario está propuesto para que los alumnos comiencen una reflexión acerca del proceso de escritura, la finalidad es que de manera consciente piensen sobre los hábitos que siguen antes y durante este proceso y, de alguna forma, también puedan identificar sus problemas; es decir, se trata de un ejercicio que incita a la autoevaluación, la metacognición y la imagen que como escritor tiene el usuario de sí mismo. Con él se pretende que los alumnos puedan reflexionar y posteriormente, con ayuda de las asesorías, reforzar o crear estrategias de escritura, es decir, "una secuencia integrada de actividades cognitivo-lingüísticas que operan como mediadoras para el logro de lo/s objetivo/s de la producción escrita de modo rápido y eficiente" (Difabio de Anglat, 2012, p. 41).

En el segundo ejercicio, a partir de diez frases los usuarios deben elaborar un párrafo que incluya al menos cuatro. Este ejercicio está propuesto para evaluar distintos rubros en el proceso de escritura:

1) Cohesión que incluye la puntuación, nexos (marcadores textuales y conjunciones), orden de elementos en la frase, uso de elementos correferenciales para evitar repeticiones léxicas y mantenimiento del referente; $y$

2) Coherencia, en la que se mide la selección de la información (que las ideas sean claras), la progresión de la información (orden lógico, tema/rema) y la estructura del texto (introducción, desarrollo y conclusión). De igual forma, en este ejercicio puede observarse la construcción y estructura del párrafo, y la selección del registro.

Además de lo anterior, en este ejercicio también puede evaluarse la ortografía que, aunque se reconoce que es una dimensión importante en el acto de escribir, sólo es un nivel más de los que hay que tener en cuenta para evaluar la adecuación de un texto.

En el tercer ejercicio un texto se encuentra desordenado por párrafos, 
el usuario debe ordenarlos para obtener un texto bien estructurado y coherente. En este ejercicio se busca medir la comprensión lectora a través de la cual los usuarios podrán relacionar el texto; al mismo tiempo, se propone evaluar la coherencia que abarca la selección de la información, la progresión de la información que engloba la introducción, la continuidad, la expansión y tropicalización (Guerra y Pérez, 2017) y, finamente, la estructura textual.

Cassany (2015) hace una distinción entre pruebas directas e indirectas, en las pruebas directas el examinado produce un texto en el que debe integrar todo tipo de elementos del sistema lingüístico, mientras que las pruebas indirectas "consisten en ejercicios de respuesta única (elección múltiple, verdadero/falso, relleno de vacíos) sobre cuestiones concretas (reglas gramaticales, vocabulario, ortografía, construcción de oraciones, uso escrito), cuyo dominio se considera imprescindible para poder producir un texto de calidad" (Cassany, 2015, p. 247). De acuerdo con esta distinción, la prueba diagnóstica que en el CE se aplica corresponde a una prueba mixta, pues es indirecta (en el ejercicio 2) y directa (en el ejercicio 3). Asimismo, esta prueba es una evaluación formativa Cassany (2015) ya que "es más cualitativa que cuantitativa, busca comprender el objeto evaluado más que medirlo, y utiliza criterios variados y adaptados a cada contexto y aprendiz" (p. 210).

La prueba antes descrita se encuentra en proceso continuo de revisión, ya que se busca mejorarla y estandarizarla hasta alcanzar el desarrollo de un instrumento diagnóstico capaz de medir las habilidades de escritura a nivel institucional, tanto cualitativa como cuantitativamente, es decir, el resultado final esperado es validar la prueba para que se convierta en una herramienta que permita detectar destrezas y debilidades de la escritura en la población universitaria. Además, como mencionan del Valle y Fuentes (2018):

Estos instrumentos, por una parte permiten elaborar un perfil de ingreso real del estudiante que se incorpora a la universidad, y por otra, usar dicha información, tanto a nivel agregado como individual, para realizar ajustes curriculares como docentes y planificar e implementar acciones de nivelación y acompañamiento para los estudiantes que lo requieran. Lo anterior de modo de que la institución se haga cargo en forma responsable y efectiva del perfil real de estudiantes que recibe y de la posible brecha entre dicho perfil y lo esperado (p.3). 
Por ello, con esta prueba, a nivel institucional, también se busca la detección de casos de estudiantes con deficiencias en la actividad de escritura para brindarles atención oportuna y en consecuencia contribuir a la reducción del rezago académico.

Dentro de los datos generales obtenidos mediante la aplicación de la prueba, se observa que la mayoría de los usuarios consideran que las dificultades que más tienen al escribir corresponden a cuestiones de coherencia, ortografía, puntuación, citación y en cómo ordenar las ideas para comenzar a redactar, existiendo una correspondencia entre estos resultados y las respuestas obtenidas en la pregunta del ejercicio uno: "¿en qué aspecto crees que puedes mejorar tu escritura?", en la que la mayoría ha descrito que en ortografía, redacción (estructurar las ideas) y puntuación.

Los usuarios que recurren al CE son generalmente estudiantes que quieren mejorar sus habilidades de escritura, ya sea porque ellos son conscientes de sus deficiencias o porque alguien más —la mayoría de las veces los profesores - los ha hecho conscientes de los errores que tienen al escribir, en ambos casos se trata de mejorar algún aspecto (o varios), la prueba de diagnóstico en buena medida ayuda a detectar cuál es el aspecto de escritura que requiere más atención. Lo anterior reafirma la idea de que "la escritura académica no es una competencia simple sino un sistema complejo de procesos interdependientes que están fuertemente vinculados a un sentimiento subyacente de autoeficacia como escritor" (Difabio de Anglat, 2012, p. 41).

Si bien los usuarios suponen cuáles son sus debilidades, aún queda por resolver la cuestión de qué factor o factores intervienen en su dificultad para escribir, en otras palabras, en qué dimensión se encuentra el problema, como mencionan Martínez y Llamas, "abunda el perfil del alumno al que se le ha indicado la oportunidad de mejorar su expresión sin mayor concreción, cuando las necesidades que cada uno presenta pueden ser de índole diversa" (2015, p. 25).

Además de lo ya mencionado, existe también la posibilidad de detectar, a través del tercer ejercicio, aquellos casos en los que las debilidades del usuario remiten a la comprensión lectora en cuanto actividad reguladora de la escritura, y no limitados únicamente a la falta de recursos de escritura. Así, también es un propósito del CE incluir en el instrumento una actividad que permita detectar las deficiencias en la comprensión lectora para poder canalizar y/o atender a los usuarios que lo requieran. 
Tabla 1

Descripción de los ejercicios que componen la prueba diagnóstica aplicada en el Centro de Escritura de la UMSNH

Ejercicios de la prueba diagnóstica aplicada en el Centro de Escritura de la UMSNH

\begin{tabular}{|c|c|c|c|}
\hline Ejercicios & $\begin{array}{l}\text { Descriptores/Criterios de Evalua- } \\
\text { ción }\end{array}$ & $\begin{array}{l}\text { Tipo de } \\
\text { respuesta }\end{array}$ & $\begin{array}{l}\text { Nivel de } \\
\text { Evaluación }\end{array}$ \\
\hline Ejercicio 1 & $\begin{array}{l}\text { Reflexión en torno al proceso de } \\
\text { escritura }\end{array}$ & \multirow[t]{2}{*}{ Abierta } & \multirow[t]{2}{*}{-} \\
\hline $\begin{array}{l}\text { Cuestionario } \\
\text { (conformado por } \\
\text { siete preguntas) }\end{array}$ & $\begin{array}{l}\text { Autoevaluación } \\
\text { Imagen que como escritor tiene } \\
\text { el usuario de sí mismo }\end{array}$ & & \\
\hline $\begin{array}{l}\text { Ejercicio } 2 \\
\text { Redacción de un } \\
\text { Párrafo }\end{array}$ & $\begin{array}{l}\text { Cohesión } \\
\text {-Orden de elementos en la frase } \\
\text { (estructura sintáctica) } \\
\text {-Mantenimiento del referente } \\
\text {-Puntuación } \\
\text {-Nexos Marcadores textuales y } \\
\text { conjunciones }\end{array}$ & \multirow[t]{2}{*}{ Abierta } & \multirow[t]{2}{*}{$\begin{array}{l}\text { Logrado/ } \\
\text { Medio } \\
\text { logrado/No } \\
\text { logrado }\end{array}$} \\
\hline & $\begin{array}{l}\text { Coherencia } \\
\text {-Selección de la información } \\
\text {-Progresión de la información } \\
\text { (orden lógico, tema/rema) } \\
\text {-Estructura del texto (Introducción, } \\
\text { desarrollo y conclusión) } \\
\text {-Selección del registro } \\
\text { Ortografía }\end{array}$ & & \\
\hline Ejercicio 3 & $\begin{array}{l}\text { Comprensión lectora } \\
\text { Coherencia }\end{array}$ & \multirow[t]{2}{*}{ Cerrada } & \multirow[t]{2}{*}{$\begin{array}{l}\text { Logrado/ } \\
\text { No logrado }\end{array}$} \\
\hline $\begin{array}{l}\text { Ordenar los } \\
\text { párrafos de un } \\
\text { texto }\end{array}$ & $\begin{array}{l}\text {-Selección de la información } \\
\text {-Progresión de la información }\end{array}$ & & \\
\hline
\end{tabular}

Actualmente, a partir de los datos obtenidos, se está trabajando en la mejora continua de este instrumento diagnóstico para identificar qué tipo de actividades son las más aconsejables para mejorar las habilidades de escritura de los usuarios. Asimismo, dado el contexto tan heterogéneo de usuarios que asisten al CE de la UMSNH, y con la intención de crear un instrumento diagnóstico que tenga un alcance institucional, 
se ha reflexionado sobre dos posibilidades, por un lado, conservar el mismo formato de la prueba pero ajustar los textos que en ella aparecen a las diferentes áreas de conocimiento; por otro lado, podría aplicarse la misma prueba a usuarios de diferentes disciplinas y grados (como se ha hecho hasta ahora) pero medir los resultados en parámetros acordes a las distintas áreas del conocimiento, sin embargo, esta es una propuesta que aún está por explorarse.

\section{Resultados y discusión}

En esta primera etapa la muestra comprende 37 pruebas diagnósticas aplicadas durante las asesorías individuales a estudiantes de diferentes grados (licenciatura y posgrado) y provenientes de diferentes áreas del conocimiento como: Ciencias Sociales y Humanidades (Historia, Letras y Derecho), Ciencias Biológicas y Agropecuarias (Biología y Veterinaria), Ciencias Económico Administrativas (Economía), Ciencias Exactas en Ingenierías (Ingeniería Química) y Arte, Arquitectura y Diseño (Arquitectura y Bellas Artes).

A partir de esta muestra se obtuvieron los siguientes resultados: en el ejercicio uno, como puede observarse en la figura 1, las respuestas más frecuentes a las preguntas "¿Escribes frecuentemente?" y "¿Qué tipo de textos?" fueron positivas, la mayoría de los estudiantes respondieron que sí escriben con frecuencia y principalmente escriben textos escolares. Sin embargo, llama la atención que la segunda respuesta más frecuente es "no, sólo textos escolares", es decir algunos estudiantes relacionan la escritura sólo al ámbito literario, considerando que la elaboración de textos escolares (académico-científicos) queda fuera del ámbito de la actividad de escribir; esta idea puede acentuarse en la respuesta "sí, textos literarios" en la que los estudiantes nuevamente relacionan las habilidades de escritura sólo a la realización de textos de creación literaria. Tal como menciona Cassany (2015), el binomio escritura y literatura se reduce erróneamente a una actividad artística, de esta manera "la representación de lo escrito como humanístico o literario ofrece un panorama sesgado de la realidad, al negar la naturaleza verbal de las ciencias" (Cassany, 2015, p. 93); así, hay todavía en algunos estudiantes una conceptualización de lo escrito como un hecho artístico y acientífico. 


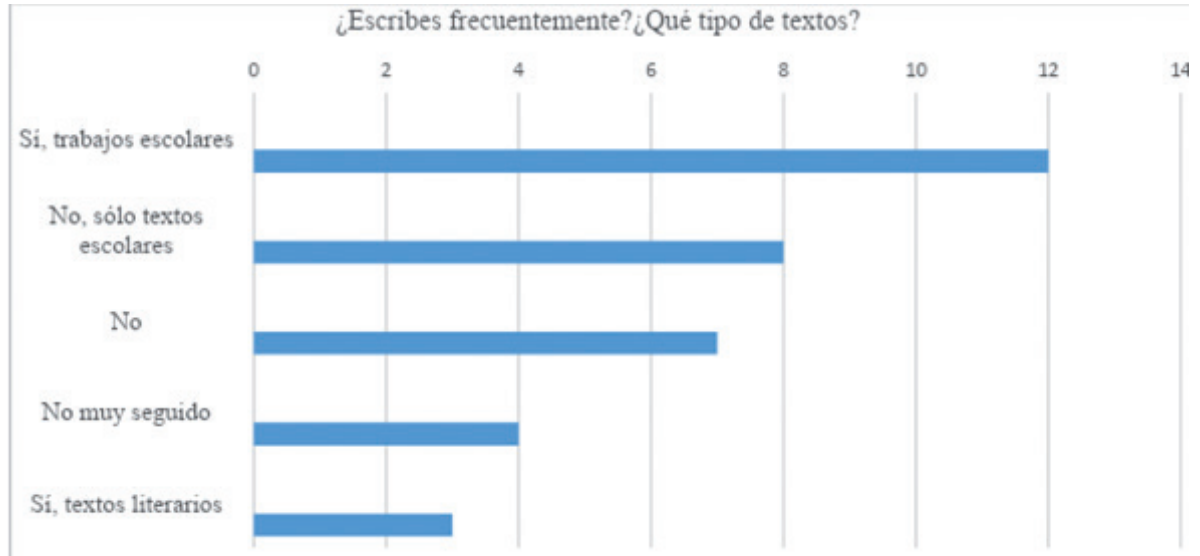

Figura 1. Frecuencia y tipos de textos que escriben los estudiantes que acuden al CE

En la pregunta número cinco del ejercicio uno, “¿Qué es lo que más se te dificulta al escribir un trabajo escolar?", las principales respuestas que se obtuvieron son: "lograr que el texto tenga coherencia", "organizar las ideas" y "cómo comenzar el texto", siendo la coherencia el rubro con mayor frecuencia como se muestra en la figura 2. Otras respuestas frecuentes fueron "cómo citar" y "la ortografía", siendo la referenciación de las fuentes, la inclusión del discurso referido y el seguimiento de reglas ortográficas, elementos que representan cierto grado de dificultad en la elaboración de un texto académico.

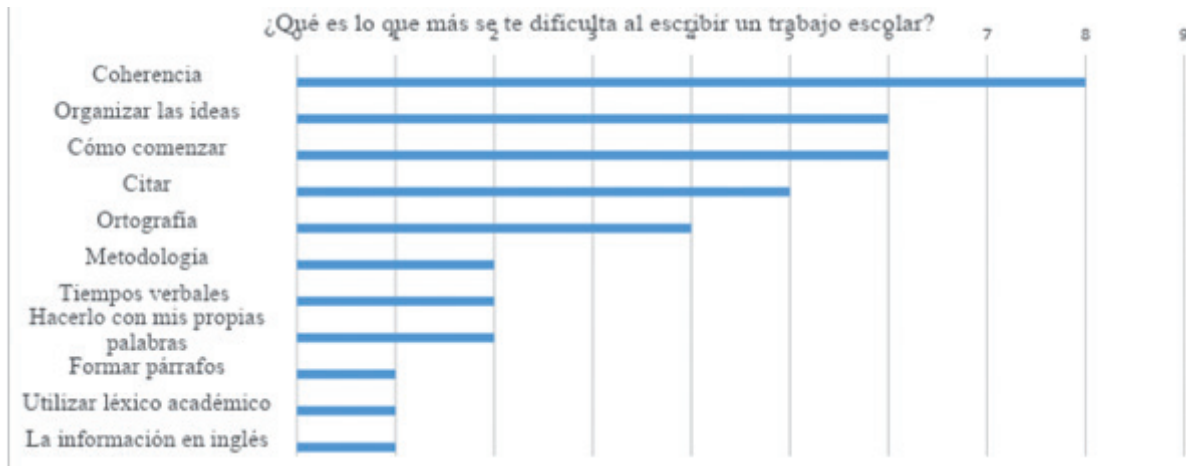

Figura 2. Principales dificultades que presentan los estudiantes al escribir un trabajo escolar

La figura 3 muestra un resumen de las principales respuestas obtenidas en la pregunta número siete del ejercicio uno: "¿En qué aspectos crees que puedes mejorar tu escritura?", en la que la principal respuesta 
fue que "en redacción" sin especificar qué aspectos de la redacción. Asimismo, es una respuesta constante "que el texto tenga coherencia" y por ende "que las ideas estén bien organizadas". Otro aspecto frecuente en el que los estudiantes consideran que pueden mejorar sus habilidades de escritura es en "tener buena ortografía".

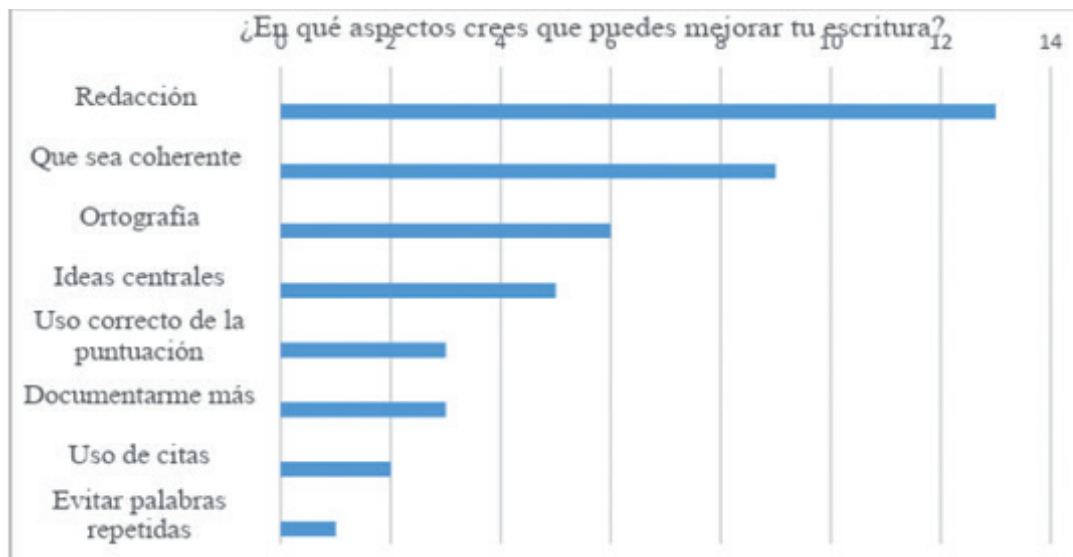

Figura 3. Aspectos que los estudiantes consideran que deben corregir para mejorar su escritura

Como puede observarse, a través de los resultados obtenidos, entre las respuestas de la pregunta 5, “¿Qué es lo que más se te dificulta al escribir un trabajo escolar?”, y la pregunta 7, “¿En qué aspectos crees que puedes mejorar tu escritura?", hay una correlación entre las características principales que los estudiantes consideran son importantes en el quehacer de la escritura, de esta manera las habilidades que buscan desarrollar son aquellas que permitan que un texto sea coherente, tener un buen manejo de la ortografía y cómo comenzar a redactar; este último es un aspecto importante, pues evidencia los esfuerzos y requerimientos cognitivos implicados en el acto de escribir, como mencionan Guerra y Pérez (2017), es una afirmación frecuente de los estudiantes: "Sé que quiero decir, pero no sé cómo decirlo" (p. 168) ante la tarea de comenzar a redactar un texto. De igual forma, estas dificultades pueden estar relacionadas a la falta de planificación del texto y a la poca claridad de los objetivos que persigue el producto textual.

Asimismo, es interesante observar que hay una sobrevaloración de la ortografía, si bien es un aspecto importante en la escritura, se trata de un elemento superficial en el sistema global de comunicación escrita. 
Algunos estudiantes consideran que un texto bien escrito en términos ortográficos es suficiente para que sea considerado un buen texto, atribuyendo más importancia a la aplicación de las reglas ortográficas que a la elaboración de un texto con significado claro, cuyo propósito sea efectivamente comunicado, esto se debe a que la educación básica en lengua materna se centra principalmente en los aspectos de ortografía y puntuación (Guerra y Pérez, 2017). En suma, en algunos casos en la escritura académica "lo primero que se valora es lo formal, después el contenido y al final lo discursivo" (Cassany, 2015, p.103).

Tal como se observa en la tabla 2, en el ejercicio número dos en el que los estudiantes tienen que redactar un párrafo, sólo 15 estudiantes obtuvieron el nivel de logrado, considerando que estructuraron un párrafo siguiendo las instrucciones del ejercicio y relacionando de manera adecuada los enunciados que eligieron para ello; además de lo anterior, se considera logrado ya que hay uso de conectores, las ideas están relacionadas y presentan una progresión temática, por lo que son textos que cumplen con las propiedades textuales de cohesión y coherencia y, por tanto, con el nivel de producción escrita que en este ejercicio se espera de los estudiantes.

Por otro lado, 14 estudiantes obtuvieron el nivel de medio logrado, entre las causas frecuentes se encuentran la ausencia del uso de conectores, la falta de relación coherente entre las ideas, la omisión del referente y la repetición de palabras. Finalmente, 8 estudiantes no lograron resolver adecuadamente el ejercicio por dos motivos, algunos estudiantes no contestaron el ejercicio y los que lo contestaron no construyeron un párrafo, sólo copiaron los enunciados, pero sin que entre éstos se estableciera alguna relación; este resultado permite observar que hay un escaso dominio de la construcción lógica de un texto, además de la falta de reconocimiento de la idea central a partir de la cual debe estructurarse.

En el ejercicio número 3 (de respuesta cerrada) entre las opciones de logrado y no logrado, predominó el nivel de logrado en 24 pruebas, mientras que 13 estudiantes no lograron ordenar los párrafos del texto de manera adecuada; en estas trece pruebas se observa que los estudiantes lograron identificar los párrafos de inicio y final del texto, siendo los párrafos de desarrollo, intermedios en el texto, los que presentaron confusión para su acomodo. 
Tabla 2

Resultados obtenidos en los ejercicios 2 y 3

\begin{tabular}{cccc}
\hline Ejercicios & Logrado & Medio Logrado & No logrado \\
\hline $\begin{array}{c}\text { Ejercicio 1 } \\
\text { Cuestionario (conformado por } \\
\text { siete preguntas) }\end{array}$ & & & \\
\hline $\begin{array}{c}\text { Ejercicio 2 } \\
\text { Redacción de un párrafo }\end{array}$ & 15 & 14 & 8 \\
\hline $\begin{array}{c}\text { Ejercicio 3 } \\
\text { Ordenar los párrafos de un texto }\end{array}$ & 24 & - & 13 \\
\hline
\end{tabular}

\section{Conclusiones}

Se comprende que no existen exámenes o actividades con parámetros universales capaces de medir las habilidades de escritura de todos los estudiantes, en todos los contextos; también se tiene consciencia de que cada aprendiz requiere de criterios de evaluación particulares, sin embargo, en el CE de la UMSNH, debido al contexto heterogéneo de usuarios, se intenta estandarizar una prueba que, junto con el borrador que cada usuario trae consigo a la asesoría, permita hacer más eficiente el desarrollo de herramientas en las habilidades de escritura y promover la autorregulación, en la que intervienen la metacognición, la cognición y la motivación (Sánchez y Martínez, 2020; Difabio de Anglat, 2012) . De esta manera, se evalúa la actividad escritora en general a través de la prueba (habilidades, conocimientos, actitudes) y la actividad escritora para una situación comunicativa específica mediante los textos que desean desarrollar o revisar los usuarios en las asesorías (uso escrito en un contexto particular), esta última actividad engloba tanto el producto escrito como el proceso a través del cual se construye el texto.

La creciente demanda que hay en los Centros y Programas de Escritura, y particularmente en el CE de la UMSNH, hace propicio el desarrollo de pruebas estandarizadas que permitan conocer las habilidades o destrezas y debilidades que poseen quienes acuden a asesorías, pues como menciona Castelló, existe la "necesidad de consensuar y sistematizar propuestas sostenibles, efectivas y válidas de evaluación tanto de los productos finales - textos- como de los procesos seguidos para su desarrollo" (2014, p. 361), de esta manera sistematizar un instrumento diagnóstico es uno de los retos actuales en el ámbito de la escritura 
académica en la universidad y particularmente en el ámbito del CE. Sin embargo, como se ha descrito en este trabajo, la elaboración de esta prueba para evaluar la escritura, aplicable y válida en un contexto tan heterogéneo, es una tarea que requiere de una revisión continua en la cual no se pueden esperar respuestas únicas, sino más bien el establecimiento de rutas de trabajo diferenciadas conforme las pruebas arrojan datos divergentes, provenientes de necesidades y trayectorias escolares específicas.

De los resultados obtenidos hasta ahora se pueden plantear algunas reflexiones; en primer lugar, los resultados deben tomarse con cautela, ya que es necesario ampliar la muestra así como el lapso de tiempo en que se implemente la prueba, pues los resultados conseguidos hasta ahora solo se han contrastado desde un enfoque cualitativo, no obstante, para validar la prueba es preciso un análisis cuantitativo. El reto del CE es, por tanto, validar más sólidamente el instrumento diseñado y analizar estos resultados tanto cualitativa, como cuantitativamente ya que para los fines que se persiguen, las evaluaciones deben mostrar una validez sistemática y confiabilidad (Sánchez y Martínez, 2020).

En segundo lugar, estandarizar una prueba también supone establecer los parámetros a través de los cuales se miden las habilidades y debilidades de escritura, por lo que es necesario mejorar la prueba diagnóstica en este rubro, este hecho implica la revisión de varias actividades que incluyen: determinar los objetivos, revisar y escoger los ejercicios más apropiados, revisar las instrucciones y las preguntas, y establecer los baremos; así mismo, analizar los resultados.

Además de lo anterior, establecer los parámetros implica delimitar las características que debe cumplir el usuario en el proceso de escritura para considerar que desarrolla bien esta actividad, es decir, se trata de medir el nivel de alfabetización académica que se supone debe ser alcanzado por los estudiantes de acuerdo a su grado, así por ejemplo, es necesario establecer los parámetros de las habilidades y destrezas de escritura que los usuarios deben poseer a nivel de pregrado y posgrado, por consiguiente es preciso responder a la cuestión ¿qué características debe reunir el escritor en un nivel formativo determinado (pregrado y posgrado) para considerar que ha alcanzado un nivel de suficiencia en la alfabetización académica que le permita desarrollarse en el ámbito universitario?

Finalmente, las habilidades de escritura pueden ser medidas a tra- 
vés de diferentes ejercicios, sin embargo, es necesario puntualizar: ¿qué criterios son los más importantes de evaluar en el proceso de escritura?, ¿Cómo evaluar sistemáticamente las habilidades de escritura en un contexto tan diverso como es el caso de la UMSNH en el que acuden al CE estudiantes de diferentes disciplinas y niveles? y ¿cuáles son los parámetros que deben seguirse en la educación superior para determinar si un estudiante es o no competente en la actividad de escritura?

\section{Referencias}

Bitonte, M. E. y Lo Coco, M. (2013). Recorridos y Actividades para la práctica de la lectura y la escritura en la educación superior. Argentina: UNM Editora.

Carlino, P. (2005). Escribir, leer y aprender en la universidad. Una introducción a la alfabetización académica. Buenos Aires: FCE.

Cassany, D. (2007). Reparar la escritura (12 a ed.). Barcelona: Editorial Graó.

Cassany, D. (2015). Construir la escritura (10 $10^{a}$ ed.). Barcelona: Paidós.

Castelló, M. (2014). Los retos actuales de la alfabetización académica: estado de la cuestión y últimas investigaciones. Enunciación, 19 (2), 346-365.

Del Valle, R. y Fuentes, C. (2018). Prueba diagnóstico de la producción escrita como herramienta de gestión académica para la permanencia y el avance curricular. En Octava Conferencia Latinoamericana sobre el abandono en la educación superior. Panamá.

Difabio de Anglat, H. (2012). Hacia un inventario de escritura académica en el Posgrado. Revista Orientación Educacional, 26(49), 37-53.

Guerra, E. y Pérez, Ma. (2017). Evaluación de la escritura en el medio universitario. En A. Enríquez y E. Sánchez (Coords.), Dominio del español de estudiantes universitarios. Retos y perspectivas (pp. 161-186). México: UMSNH.

Guzmán-Simón, F. y García-Jiménez, E. (2015). La evaluación de la alfabetización académica. RELIEVE, 21 (1), 1-25. DOI: 10.7203/relieve.21.1.5147

Martínez, C. y Llamas C. (2015). Implantación del Centro de Escritura en la Universidad de Navarra: líneas generales y pruebas de diagnóstico. En I. Ballano e I. Muñoz (Eds.), La escritura académica en las universidades españolas (pp. 11-40). Bilbao: Publicaciones de la Universidad de Deusto.

Molina, V. (2019). El discurso pedagógico en las tutorías de escritura. Develando elementos de una práctica educativa. Revista mexicana de investigación educativa, 24 (80), 125-148.

Roldan, Y., Cárdenas C. y Casanova R. (2013). Teoría y práctica de la escritura académica. Chile: Ediciones Universidad Austral de Chile.

Sánchez, M. y Martínez A. (2020). Evaluación del y para el aprendizaje: instrumentos y estrategias. México: UNAM. 